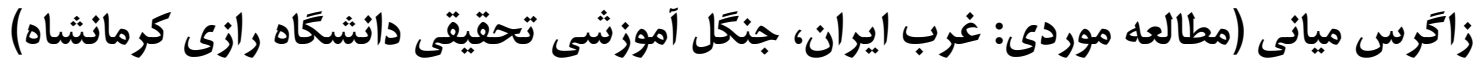

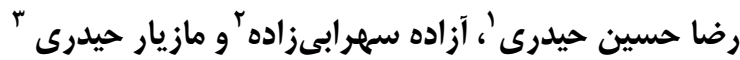

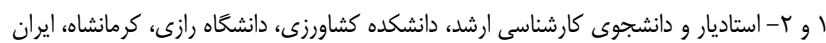 \\ ب- استاديار يزوهش، بخش تحقيقات جنگل ها و مراتع، مركز تحقيقات و آموزش كشاورزى و و منابع طبيعى استان كردستان، سنندج، ايران،

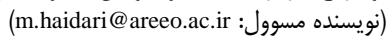

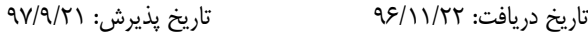 \\ Vف Vفه:
}

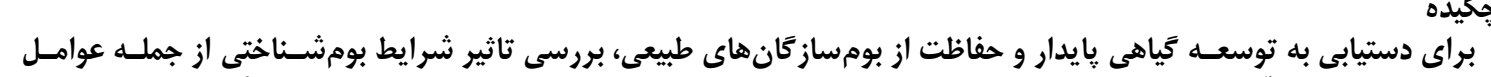

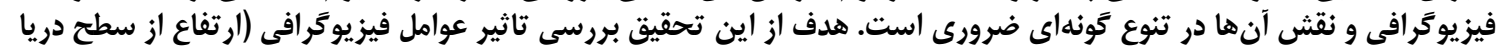

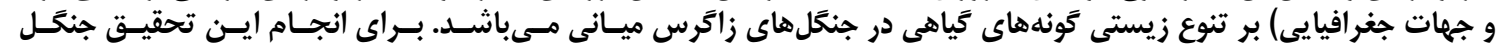

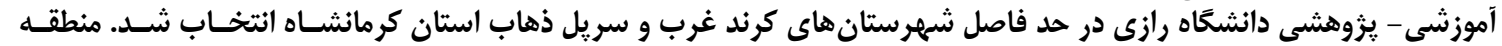

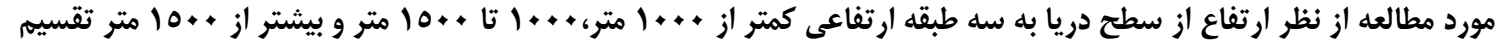

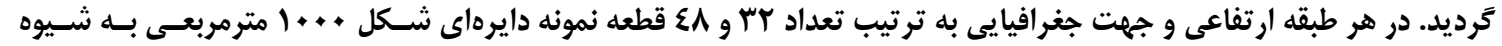

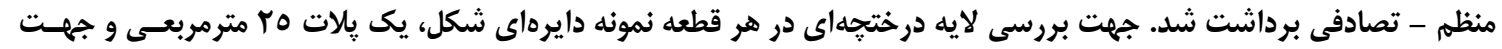

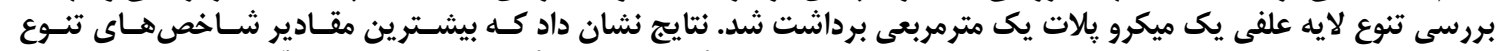

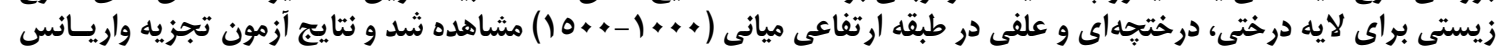

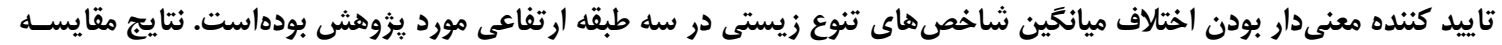

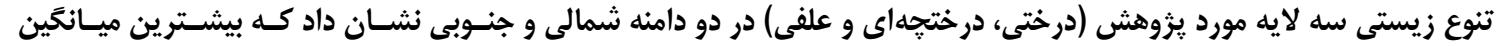

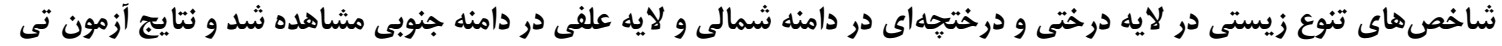

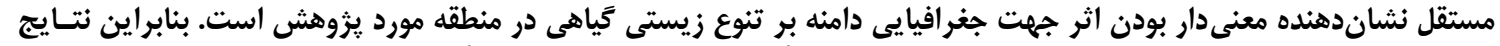

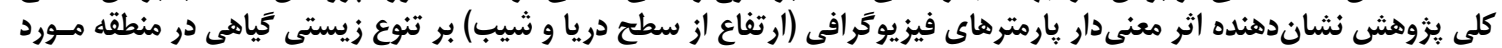
ئزوهش است.

وازههاى كليدى: شاخص هاى تنوع زيستى، قطعه نمونه، طبقه ارتفاعى، ميكرو بِلات

ويوجينوويك و همكاران (rآ) ارتفاع از سطح دريا، جهت

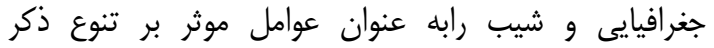

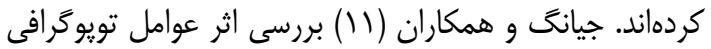

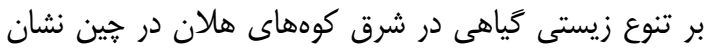

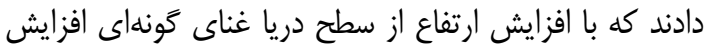

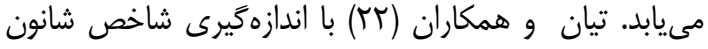

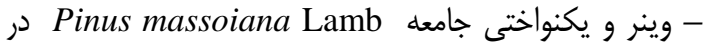
يروزه حفاظت از تنوعزيستى در ناحيه حفاظت شأل شده در يانكتاز

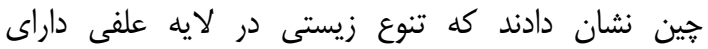

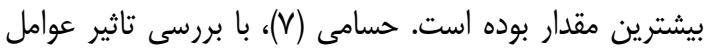

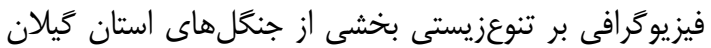

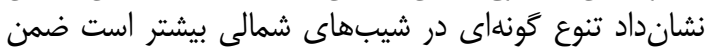

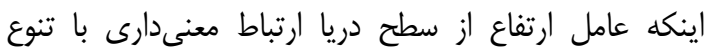

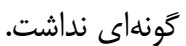

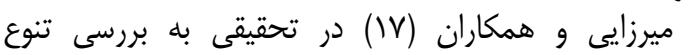

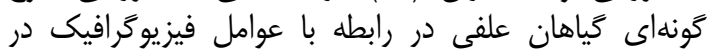

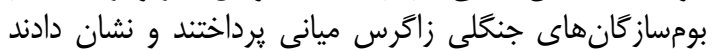

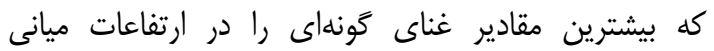

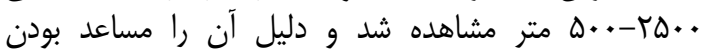

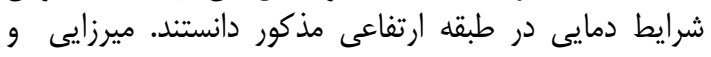

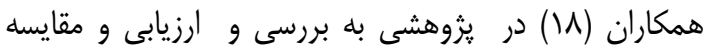

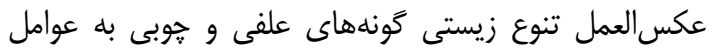

زاكرس سلسله جبالى است كه از شمال غربى به طرف

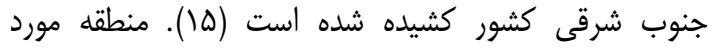

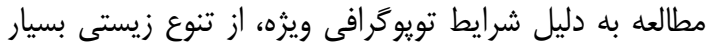

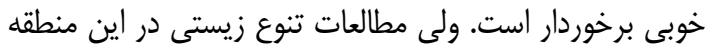

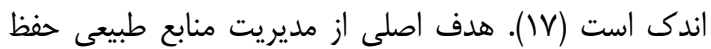

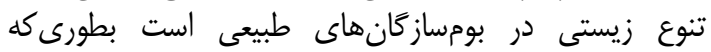

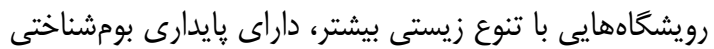

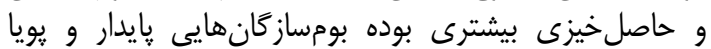

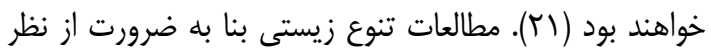

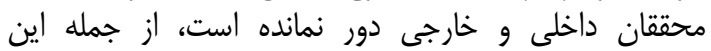
تحقيقات مى توان به موراد زير اشاره كرد إنان

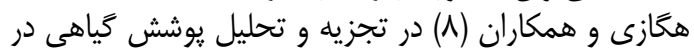

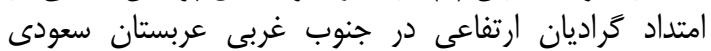

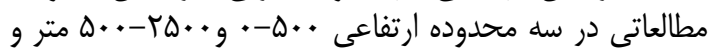

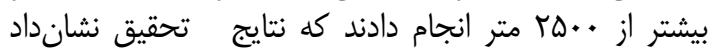

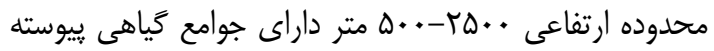

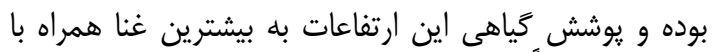

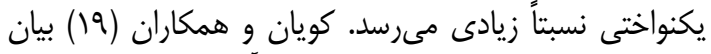

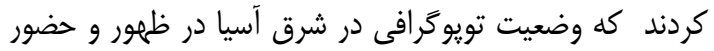

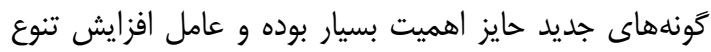
زيستى آن منطقه در مقايسه با جنوب شرقى آمريكاست 
سطح دريا و جهت دامنه بر تنوع زيستى گياهان در كوهستانهاى غرب استان كرمانشاه (در گيلانغرب) تيرداختند

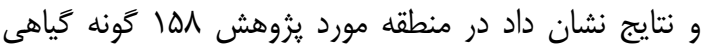

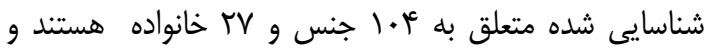

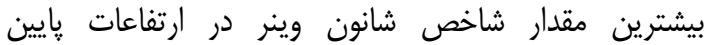

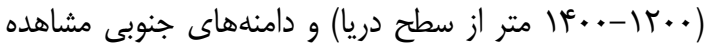

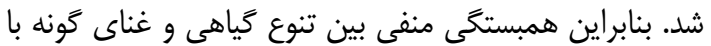

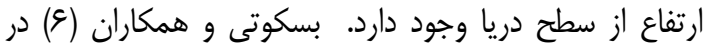

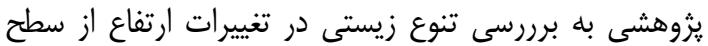

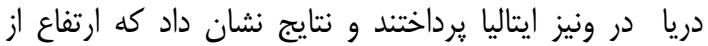

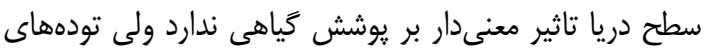

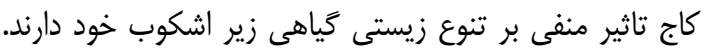

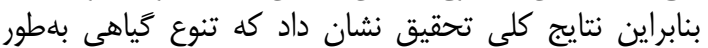

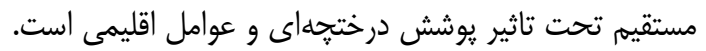

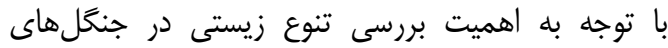

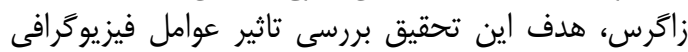

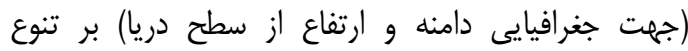

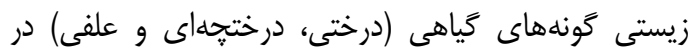

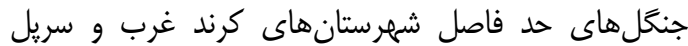
ذهاب استان كرمانشاه است.

\section{مواد و روشها

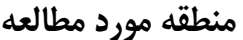

منطقه مورد مطالعه در مور حد فالعه فاصل شهرستانهاى كرند

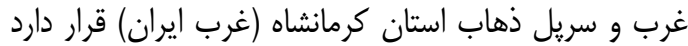

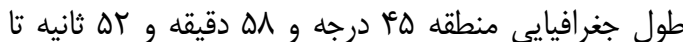

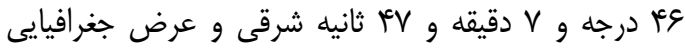

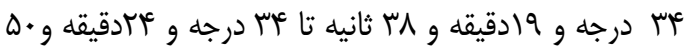

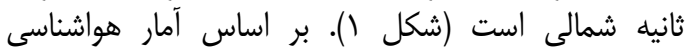

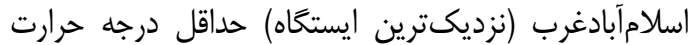

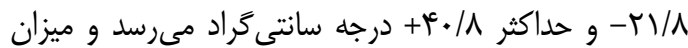

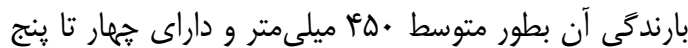

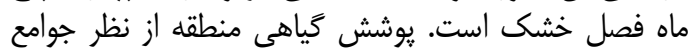

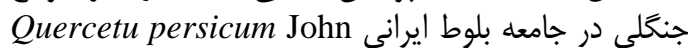

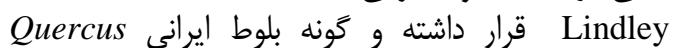

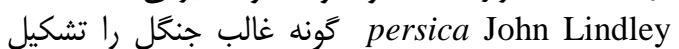

مى دهد (.).
محيطى در جهتهاى مختلف جغرافيايى جنگل إداى زاگرس

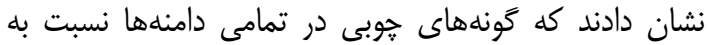

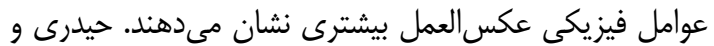

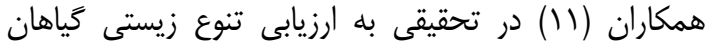

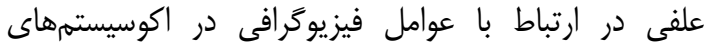

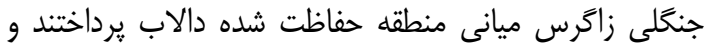

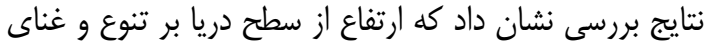

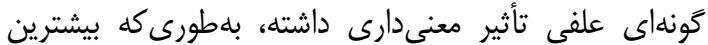

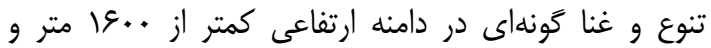

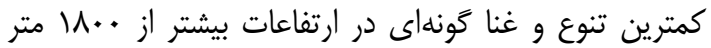

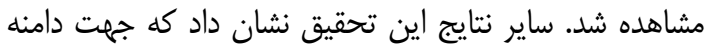

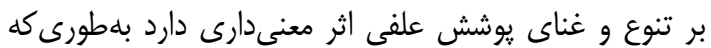

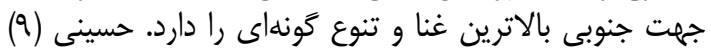

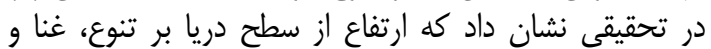

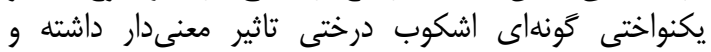

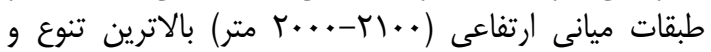

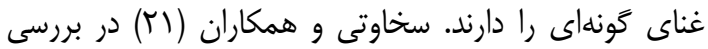

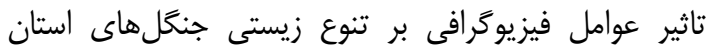

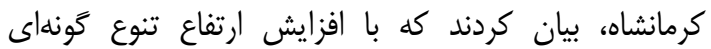

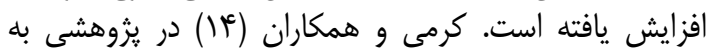

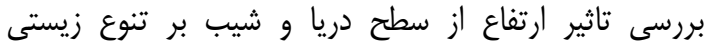

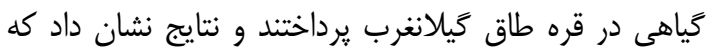

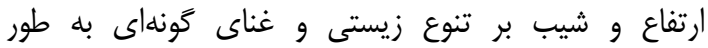

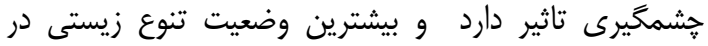

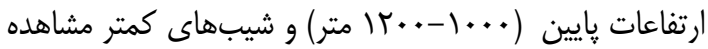

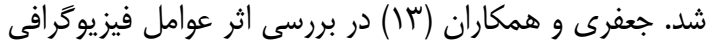

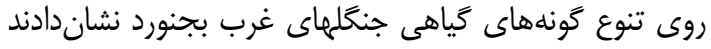

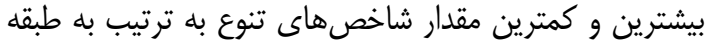

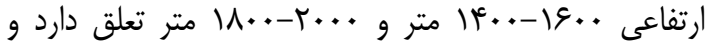

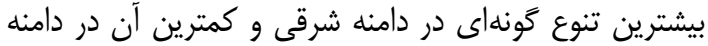

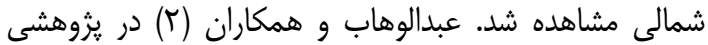

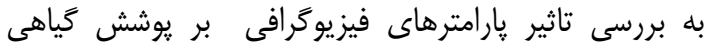

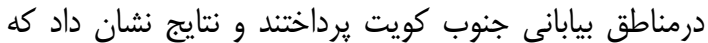

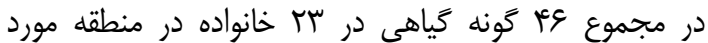

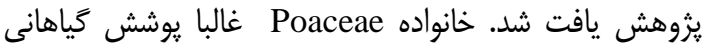

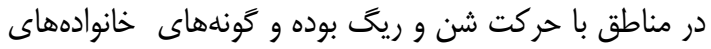
Chenopodiacea و Asteraceae قسمتهاى ساحلى تشخيص داده شدند. محمودى و و

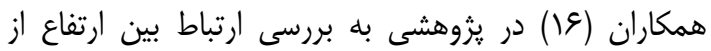




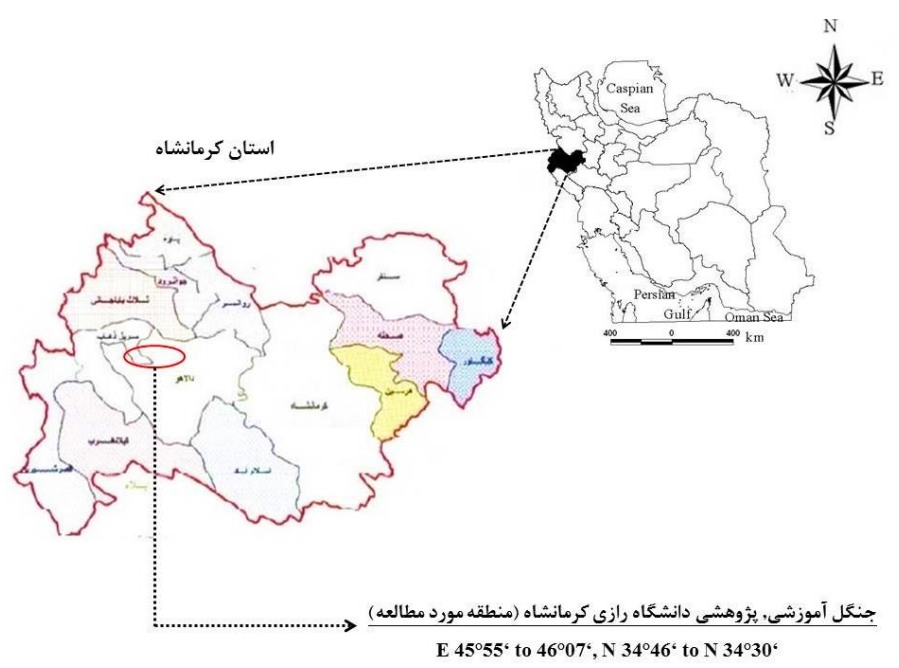

شكل (- موقعيت منطقه مورد مطالعه در ايران و استان كرمانشاه (غرب ايران)

Figure 1. Location of the studied area in Iran and Kermanshah Province (West of Iran)

قطعلنمونه در منطقه مورد مطالعه برداشت گرديد. جهت بررسى

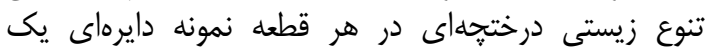

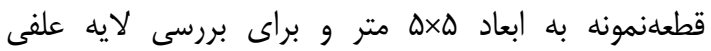

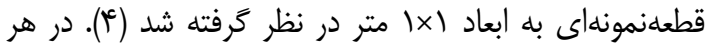

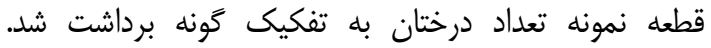

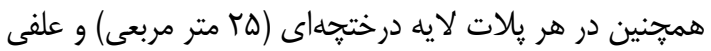

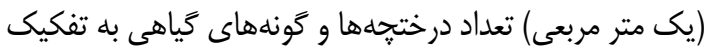

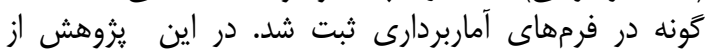

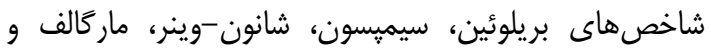

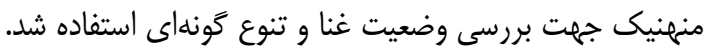
جهت بررسى تاثير ارتفاع از سطح دريا و جهت جغر جرافيايى بر

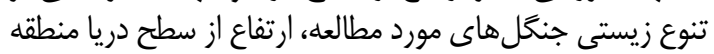

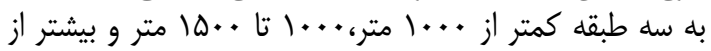

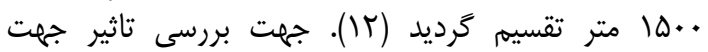

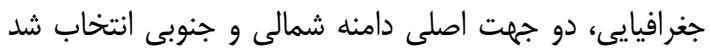

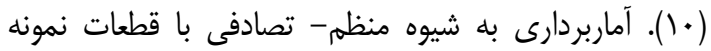

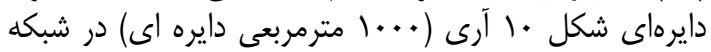

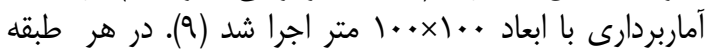

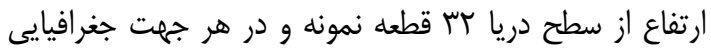

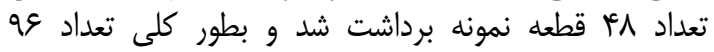

Table 1. Indicators used in this study

جدول 1- شاخصهاى مورد استفاده در اين مطالعه

\begin{tabular}{|c|c|}
\hline شاخص & فرمول \\
\hline 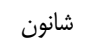 & $H^{\prime}=\sum_{i=1}^{s} p_{i} \operatorname{Ln}\left(p_{i}\right)$ \\
\hline سيميسون & $1-D=\sum\left(p_{i}\right)^{2}$ \\
\hline ماركالوف & $M=(S-1) / \operatorname{Ln}(N)$ \\
\hline بريلوئين & $\operatorname{LnN}_{i} \sum \operatorname{Lnn}_{i} / N$ \\
\hline منهنيك & $S / \sqrt{N}$ \\
\hline
\end{tabular}
فراوانى هر كونه pi pi تعداد كل كَونهاى مورد مطالعه S

م يانخين شـــاخص هاى تنوع گو نهاى در دو طب قه جهرت

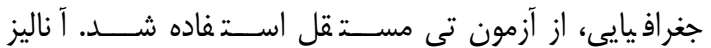

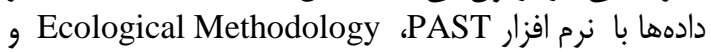

SPSS 20

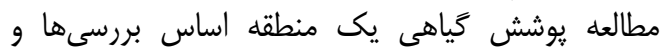

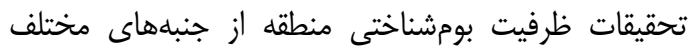

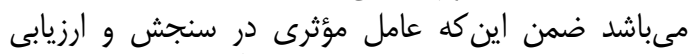

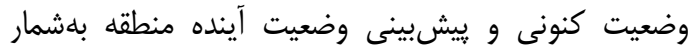
مىرود كه براى اعمال مديريت صحيح نقش بلهسز ايیى دارد
تعيين و برآورد تنوعزيســتى گيـاهى اغلـب در قـالـب شاخصهاى عددى غناى گَونهاى تعيين مى شود (19) بر بر اين

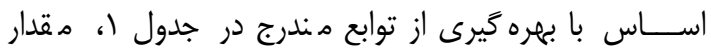

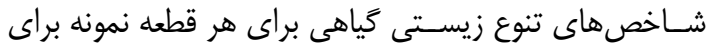

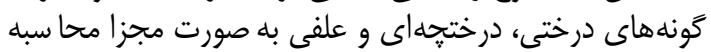

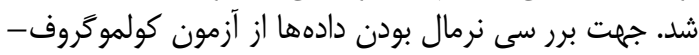

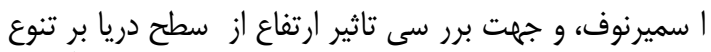

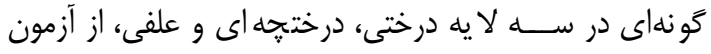

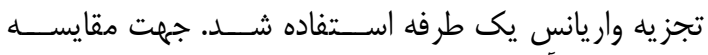
ميانخينها از آزمون دانكن استفاده شد. جهت برفي اسر رسى و مقايسه 


\section{نتايج بررسى نشان داد كه در منطقه مورد مطالعه جهار گَونه درختى و ينج گَونه درختجهاى وجود دارد (جدول ؟).}

Table 2. Characteristics of tree and shrubs species in the studied area

جدول r- مشخصات گَونهاى درختى و درختجهاى منطقه مورد مطالعه

\begin{tabular}{|c|c|c|c|c|}
\hline درختى / درختجهاى & 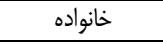 & اسم علمى & اسم فارسى & رديف \\
\hline درختى & Fagaceae & Quercus brantii John Lindley & بلوط ايرانى & 1 \\
\hline 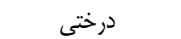 & Rosaceae & Crataegus aronia Carl Linnaeus & 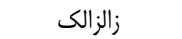 & r \\
\hline 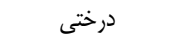 & Fagaceae & Quercus infectoria Olivier Chabaud & مازودار & r \\
\hline 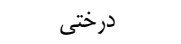 & Rosaceae & Pyrus glabra Te-tsun Yü & كَلابى وحشى & f \\
\hline درختهِ|ى & Rosaceae & Amygdalus reuteri David Allardice Webb & بادام كوهى (ارثن) & $\Delta$ \\
\hline درختهِ|ى & Sapindaceae & $\begin{array}{c}\text { A. cinerascens Thomas de Grey, 6th Baron } \\
\text { Walsingham }\end{array}$ & 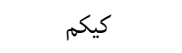 & $q$ \\
\hline درختهِ|ى & Caprifoliaceae & $\begin{array}{c}\text { Lonicera nummularifolia J. \& sp Carl } \\
\text { Linnaeus }\end{array}$ & קיاخور (شن) & $\checkmark$ \\
\hline درختجهاى & Rosaceae & Prunus cerasus & آلبالو وحشى & $\wedge$ \\
\hline درختهِ|ى & Rosaceae & $\begin{array}{c}\text { Cerasus microcarpa (C.A.M) Boiss Carl } \\
\text { Anton von Meyer }\end{array}$ & راناس (براليك) & 9 \\
\hline
\end{tabular}

كَونه) بيشترين فراوانى رادر منطقه مورد مطالعه داشتند (شكل

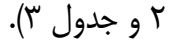

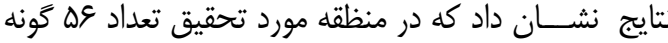

كياهى مشـاهده شــد و در كل خانوادههاى :

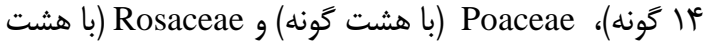

Table 3. Characteristics of plan species the Region under study

جدول س- مشخصات كَونهاى كَياهى منطقه مورد مطالعه

\begin{tabular}{|c|c|c|c|c|c|}
\hline خانواده & 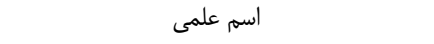 & رديف & خانواده & 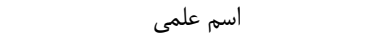 & رديف \\
\hline Apocynaceae & Nerium oleander Carl Linnaeus & rq & Poaceae & Secale Carl Linnaeus & 1 \\
\hline Aristolochiaceae & Aristolochia olivieri Collegno & r. & Chenopodiaccac & Noaea mucronata & r \\
\hline Brassicaceae & Hirschfeldia incana Carl Linnaeus & 部 & Poaceae & Aegilops triuncialis Carl Linnaeus & r \\
\hline Brassicaceae & Isatis raphanifolia Carl Linnaeus & tr & Poaceae & Bromus danthoniae Carl Linnaeus & r \\
\hline Caryophyllaceae & Dianthus orientalis Carl Linnaeus & سז & Poaceae & Hordeum marinum William Hudson & $\Delta$ \\
\hline Caryophyllaceae & Silene vivianii Carl Linnaeus & me & Poaceae & Poa bulbosa Carl Linnaeus & \& \\
\hline Chenopodiaceae & Atriplex leucoclada Carl Linnaeus & ra & Poaceae & Hordeum bulbosum Carl Linnaeus & $\checkmark$ \\
\hline Chenopodiaceae & Salsola rigida Carl Linnaeus & 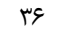 & Gramineae & Kochia prostrate (L.) A.J.Scott & $\wedge$ \\
\hline Asteraceae & Achillea vermicularis Carl Linnaeus & $r v$ & Iridaceae & Iris hymenospatha Carl Linnaeus & 9 \\
\hline Asteraceae & Achillea gillettii Carl Linnaeus & ऍی & Lamiaceae & $\begin{array}{c}\text { Salvia kermanshahensis Carl } \\
\text { Linnaeus }\end{array}$ & 1. \\
\hline Asteraceae & Centaurea behen Carl Linnaeus & rq & Lamiaceae & Stachys cretica Carl Linnaeus & 11 \\
\hline Asteraceae & Centaurea virgate Lamarck & f. & Asteraceae & $\begin{array}{c}\text { Artemisica chamaemelifolia } \\
\text { Dominique Villars }\end{array}$ & ir \\
\hline Asteraceae & Cousinia kirrindiea & (1) & Lamiaceae & Thymus kotschyanus Carl Linnaeus & Ir \\
\hline Asteraceae & Cichorium intybus Carl Linnaeus & et & Malvaceae & Alcea calverti & $1 f$ \\
\hline Asteraceae & Gundelia tournefortii Carl Linnaeus & kr & Orchidaceae & Ophrys reinholdii & 10 \\
\hline Asteraceae & Echinops ecbatanus William Roxburgh & kt & Papilionaceae & $\begin{array}{c}\text { Astragalus bruguieri Pierre Edmond } \\
\text { Boissier }\end{array}$ & 19 \\
\hline Asteraceae & Echinops haussknechtii Carl Linnaeus & ia & Papilionaceae & Lathyrus gorgoni Carl Linnaeus & IV \\
\hline Asteraceae & Helianthemum lippii Philip Miller & is & Papilionaceae & Astragalus gossypinus Carl Linnaeus & 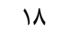 \\
\hline Asteraceae & $\begin{array}{r}\text { Heteranthelium piliferum Hochst. ex Jaub. } \\
\text { \& Spach }\end{array}$ & eV & Solanaceae & $\begin{array}{c}\text { Hyoscyamus kotschyanus Carl } \\
\text { Linnaeus }\end{array}$ & 19 \\
\hline Asteraceae & $\begin{array}{c}\text { Senecio vernalis Franz de Paula Adam von } \\
\text { Waldstein }\end{array}$ & is & Solanaceae & Hyoscyamus leptocalyx Carl Linnaeus & r. \\
\hline Compositae & Tragopogon collinus Carl Linnaeus & kq & Rhamnaceae & Paliurus spina-christi Philip Miller & rI \\
\hline Cyperaceae & Carex sp. Carl Linnaeus & Q. & Salicaceae & Populus euphratica Daniel Oliver & tr \\
\hline Euphorbiaceae & Euphorbia spp. Carl Linnaeus & Q) & Rosaceae & $\begin{array}{c}\text { Amygdalus Arabica David Allardice } \\
\text { Webb }\end{array}$ & זr \\
\hline Fabaceae & Lotus corniculatus Carl Linnaeus & QT & Rosaceae & $\begin{array}{c}\text { Amygdalus scoparia David Allardice } \\
\text { Webb }\end{array}$ & $t^{c}$ \\
\hline Fabaceae & $\begin{array}{l}\text { Onobrychis saliva Giovanni Antonio } \\
\text { Scopoli }\end{array}$ & QH & Rosaceae & $\begin{array}{c}\text { Cotoneaster nummularia Friedrich } \\
\text { Kasimir Medikus }\end{array}$ & tQ \\
\hline Fabaceae & Medicago sativa Carl Linnaeus & DF & Juncaceae & Juncus sp Carl Linnaeus & לe \\
\hline Poaceae & Bromus tectorum Carl Linnaeus & $\Delta \Delta$ & Verbebnaceae & Vitex pseudo-negundo Carl Linnaeus & tr \\
\hline Oleaceae & Dapehne mucronata Carl Linnaeus & $\Delta S$ & Zygophyllaceae & Peganum harmala Carl Linnaeus & ru \\
\hline
\end{tabular}


V. بررسى تأثير عوامل فيزيوگرافى بر تنوع زيستى گونههاى گياهى در جنكلهاى زاكرس ميانى ..

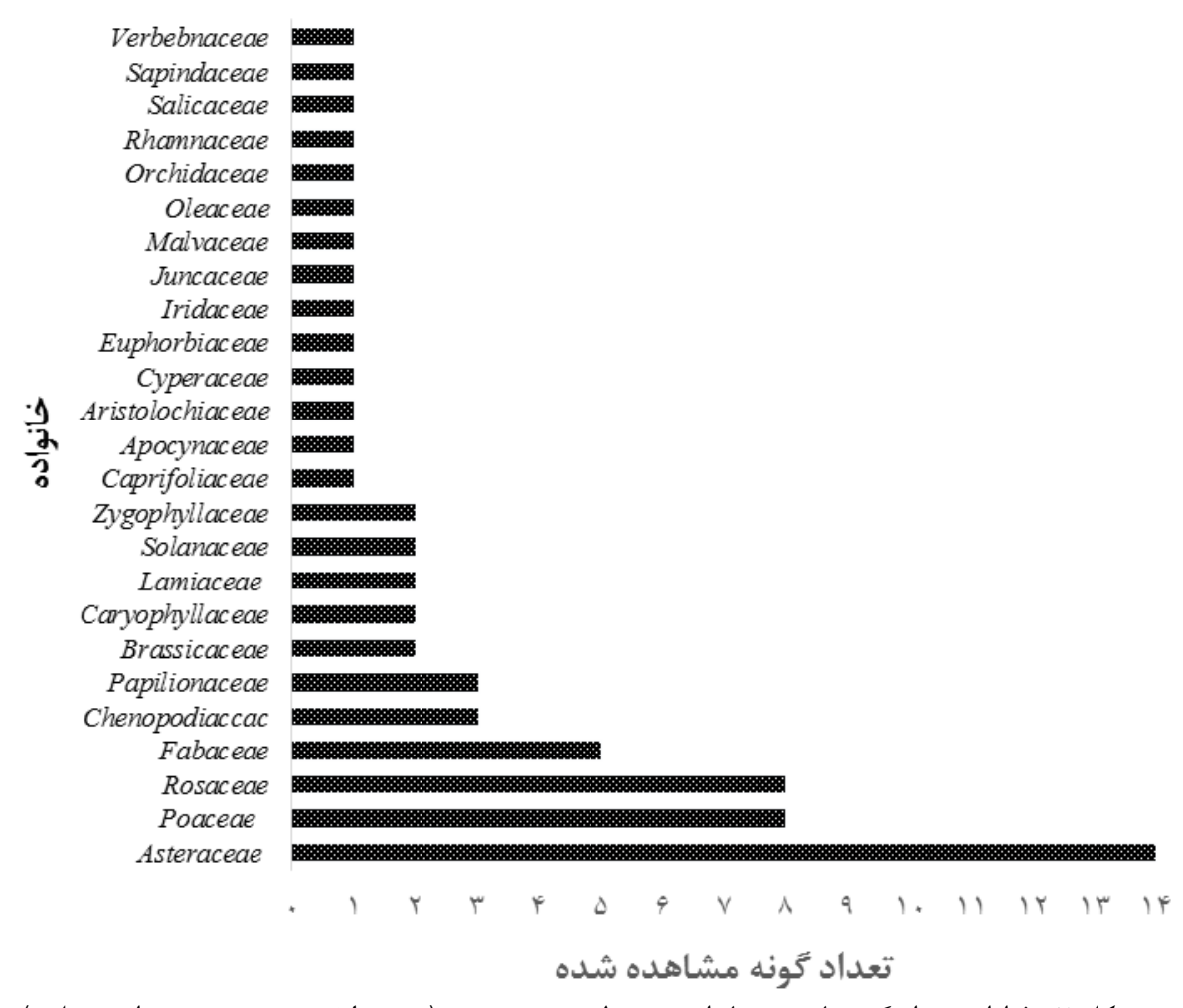

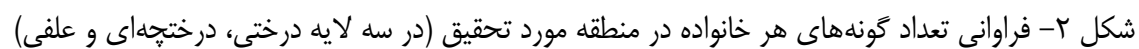

Figure 2. Frequency of number of species per family in the studied area (in three layers of tree, shrub and herbaceous)

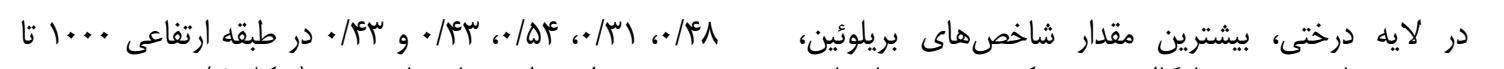

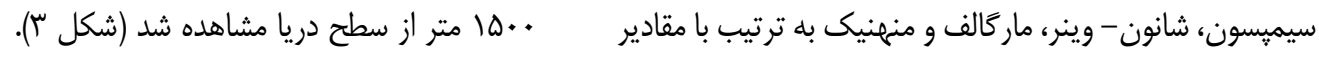

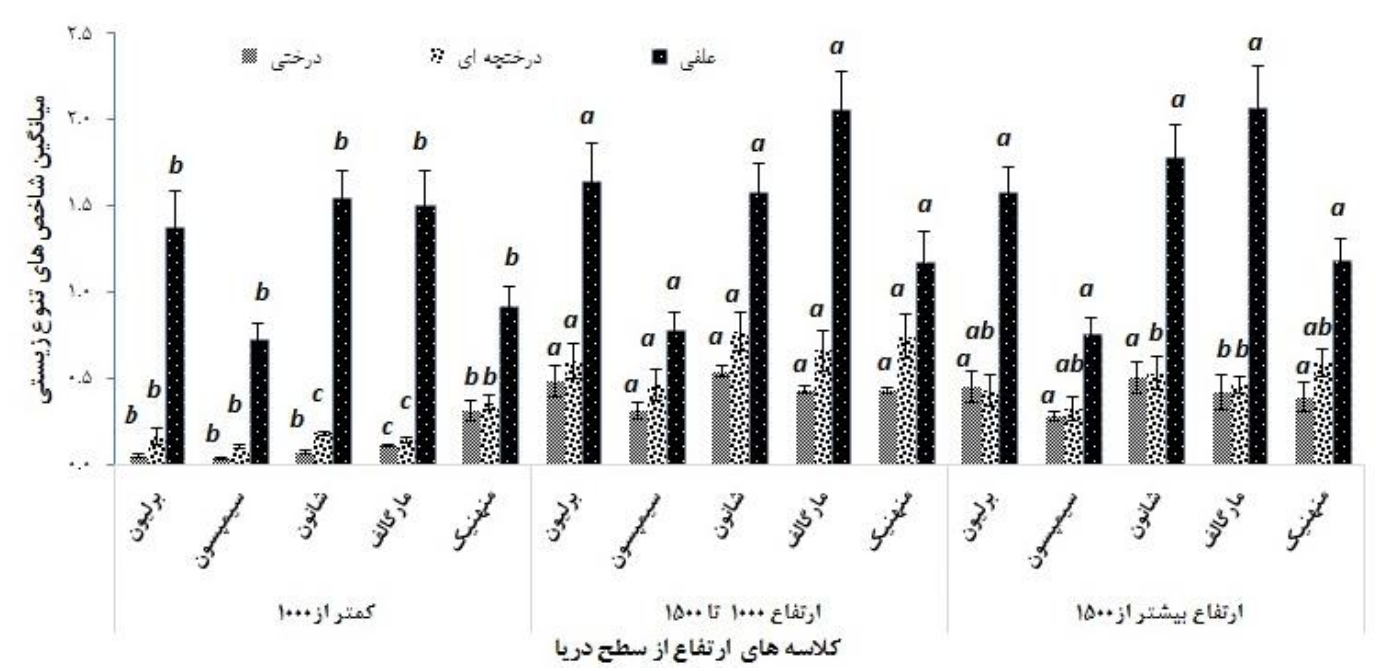

شكل س- مقايسه ميانگين شاخصهاى تتوع زيستى در سه طبقه ارتفاع از سطح دريا و لايه هاى درختى، درختهاهى و علفى با استفاده از آزمون دانكن

Figure 3. Mean comparison of biodiversity indices in three classes of altitude above sea level and tree, shrub and herbaceous layers using by Duncan test

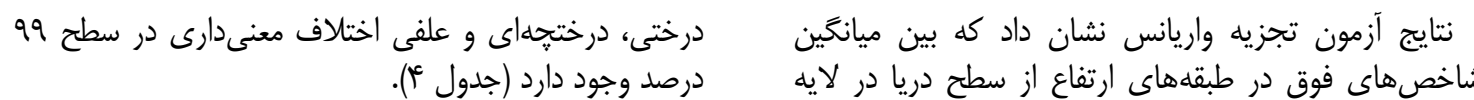




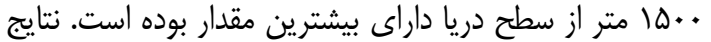

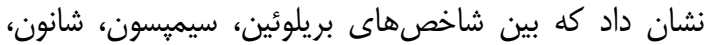
مار كالف و منهنيك در طبقهان

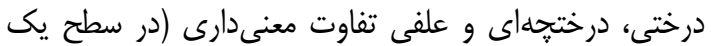

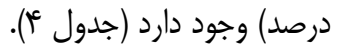

در لايههاى درختى، درختجهاى و علفى نتايج آزمون دانكن

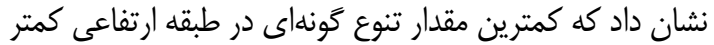

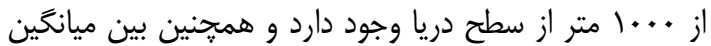

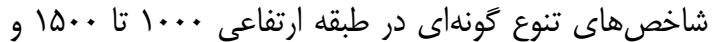

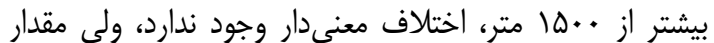

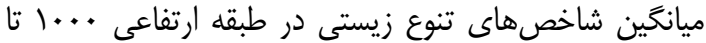

جدول ع- نتايج آزمون تجزيه واريانس جهت بررسى تاثير ارتفاع از سطح دريا بر تنوع گَونهاى درختى، درختجهاى و علفى Table 4. Analysis of variance for assessing the effect of altitude from sea level on the species diversity of the tree, shrub and herbaceous species

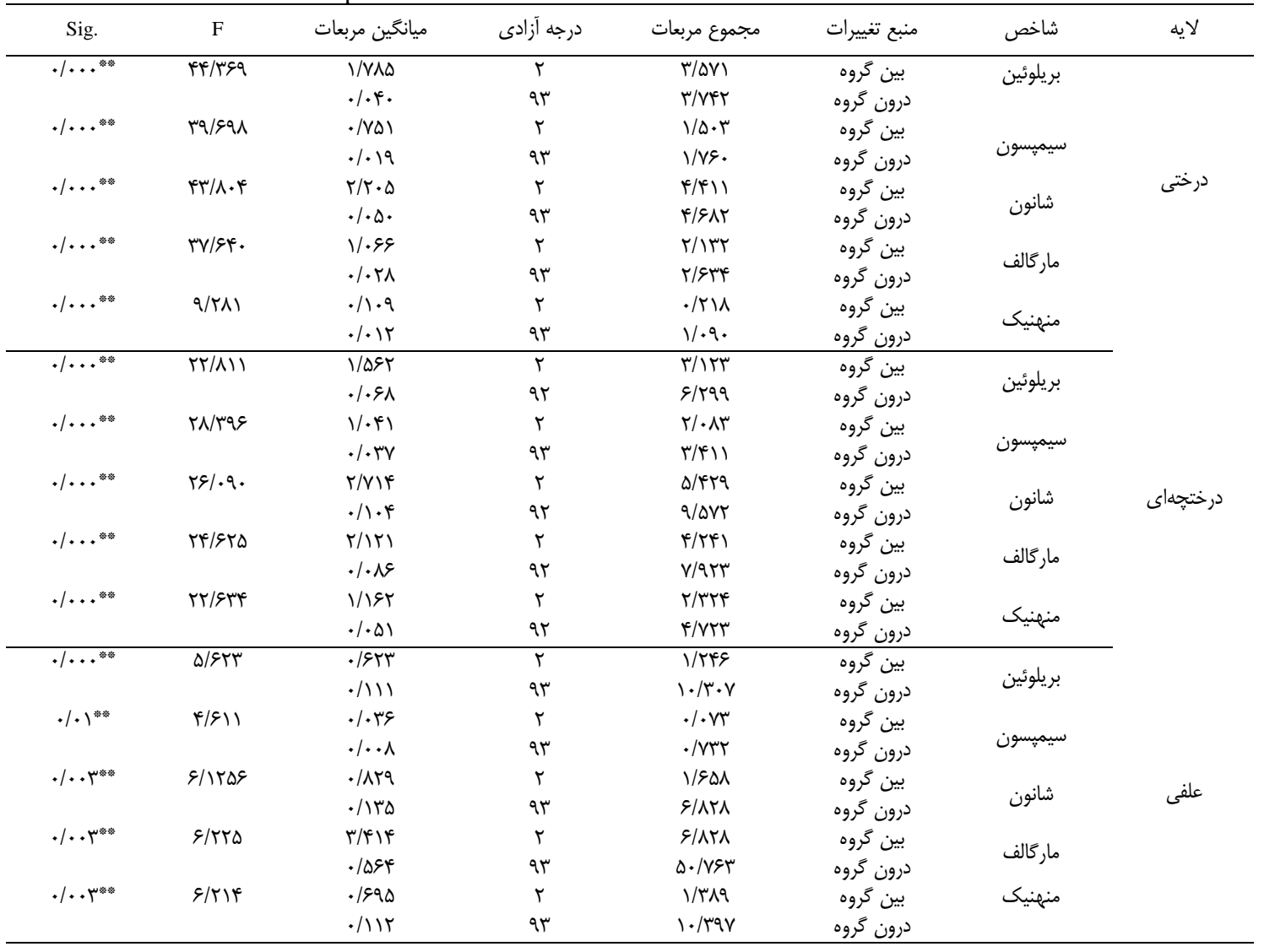

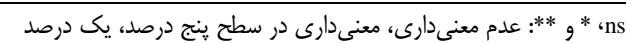

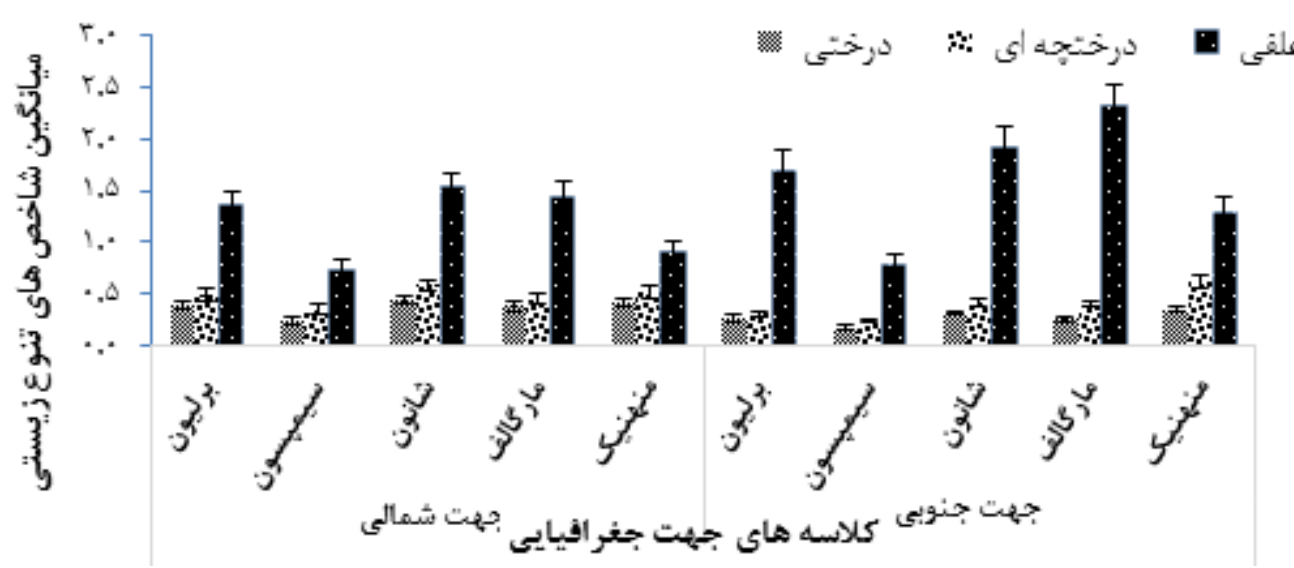

شكل عأ- مقايسه ميانگين شاخصهاى تنوع زيستى در جهات جغرافيايى شمالي و جنوبى در لايههاى درختى، درختجهاى و علفى Figure 4. Comparison of the average of biodiversity indices in directions of the geographical range northern and southern in the tree, shrub and herbaceous layers 
شاخص بريلوئين در لايه علفى در دو جهت جغرافيايى اختلاف معنى دارد وجود نداشت).

نتايج ز شان داد كه بين ميانخين شاخص شهاى تنوع گَونهاى

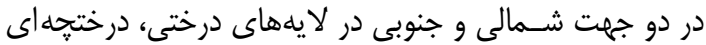

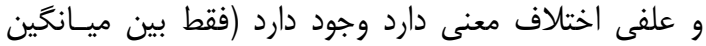

جدول ه- نتايج آزمون تى مستقل جهت بررسى تاثير جهت جغرافيايى بر تنوع گَونهاى درختى، درختجهاى و علفى Table 5. Independent T-test results for study of the effect of geographical direction on the species diversity of the tree, shrub and herbaceous species

\begin{tabular}{|c|c|c|c|c|c|c|}
\hline Sig. & $\mathrm{t}$ & Sig. & $\mathrm{F}$ & درجه آزادى & شاخص & لايه \\
\hline$\cdot / \cdot \Delta)^{\mathrm{cns}}$ & $-1 / 9 \Delta \Delta$ & $. / \Lambda \mid \wedge$ & $\cdot / \Delta \Gamma$ & 94 & بريلوئين & \\
\hline ..$q^{\mathrm{nns}}$ & $-1 / A \gamma q$ & $\cdot \mid \Lambda \& \wedge$ &.$/ . \Gamma \Lambda$ & 94 & سيميسون & \\
\hline$\cdot / \cdot t^{*}{ }^{*}$ & $-r / \cdot v q$ &.$/ 909$ & r...r & 9) & شانون & درختى \\
\hline$\cdot / . \varphi^{* * *}$ & $-r / Q I V$ & . & / /TTV & 94 & ماركالف & \\
\hline $1+\Delta^{* * *}$ & $-r / A V G$ & - $/ \Delta T^{c}$ & . & 94 & منهنيك & \\
\hline$\cdot / \cdot r^{* * *}$ & $-r / / Y T^{r}$ &.$/ \cdot r$ & $1 . / \% \cdot 9$ & 94 & بريلوئين & \\
\hline.$/ \ldots * *$ & - &.$/ \ldots$ & 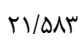 & 94 & سيميسون & \\
\hline.$/ \cdot r \Delta^{*}$ & Tאוא/T- &.$/ \ldots$ & $r .1 .9$ & $9 \%$ & شانون & درختجهاى \\
\hline$\cdot / \uparrow a \cdot n s$ & $\because / V \Delta \Lambda$ &.$/ \ldots$ & $r \Delta / \Delta \Lambda$ & 94 & مار كالف & \\
\hline$\cdot / \cdot \varphi q^{*}$ & T/.rT &.$/ \ldots$ & $r q / . r$ & 94 & منهنيك & \\
\hline$\cdot /{ }^{* * *}$ & $\Delta / T Y A$ & $\cdot / 19$ & $1 / 941$ & 94 & بريلوئين & \\
\hline$\cdot / . .1^{* *}$ & $r / F q \Lambda$ &.$/ 95$ &.$/ . r$ & 94 & سيميسون & \\
\hline.$/ \ldots * *$ & $\Delta / F^{*} V^{c}$ &.$/ r$ & Tr/א & 94 & شانون & علفى \\
\hline.$/ \ldots * *$ & $s / V)$. &.$/ \ldots$ & $M / F$ & 94 & مار كالف & \\
\hline.$/ \ldots * *$ & 91.90 & $.1 \cdot 1$ & $\varepsilon / T \Delta$ & qf & منهنيك & \\
\hline
\end{tabular}

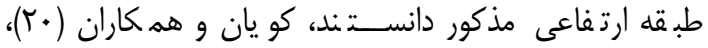

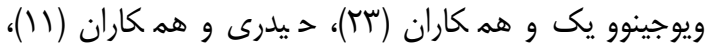

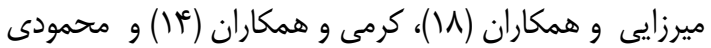

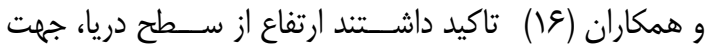

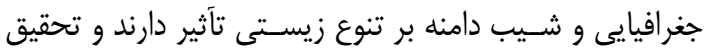

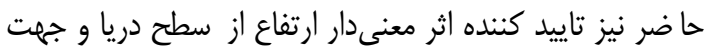

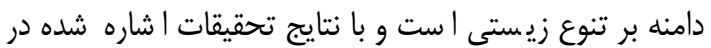

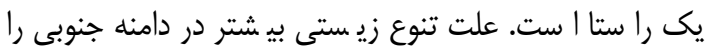

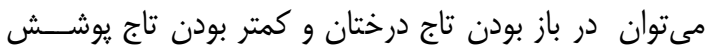

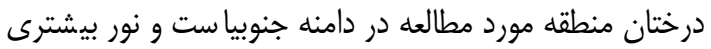

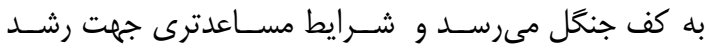

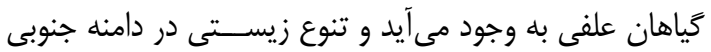

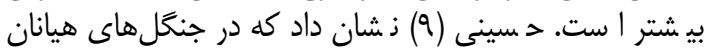

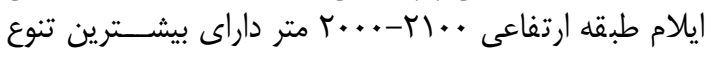

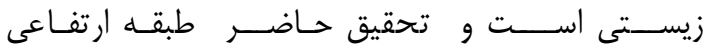

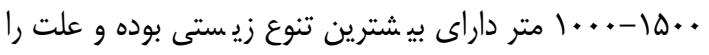

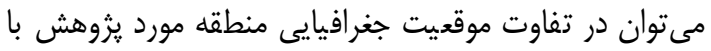

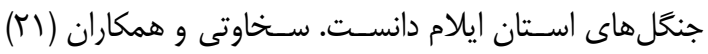

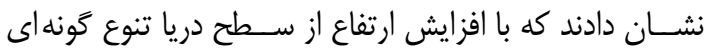

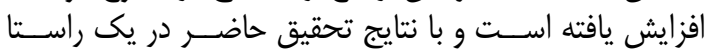

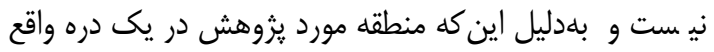

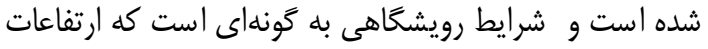

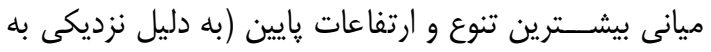
مناطق مسكونى و تخريب توسط روستائيان) و ارتفاعات ارتفات باتئ بالاتر

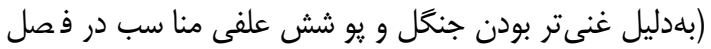

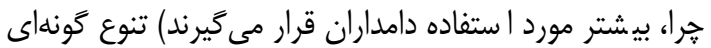

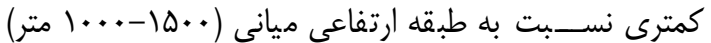

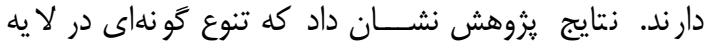

فيزيوگرافى به معنى شكل سطحى يك منطقه است كه

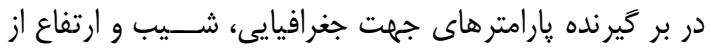

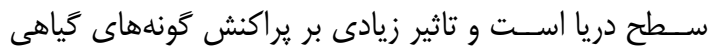

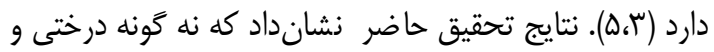

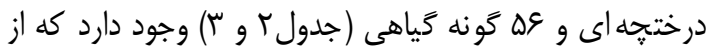
نظر فراوانى بيشترين آن متعلق به خانه (جلون آنوده

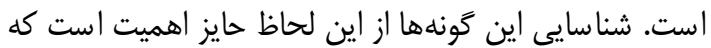

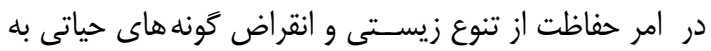

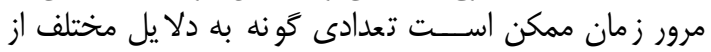

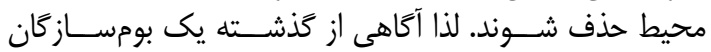

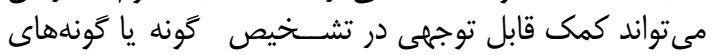

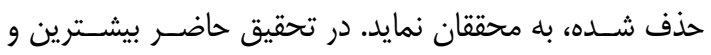

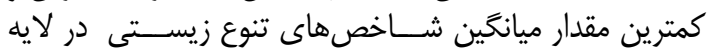

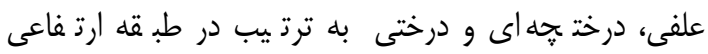

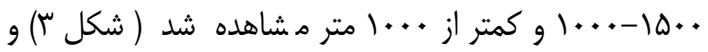

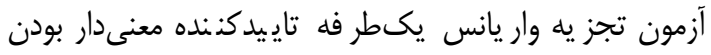

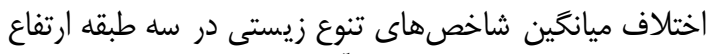

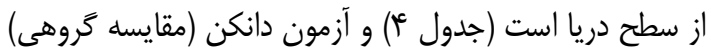

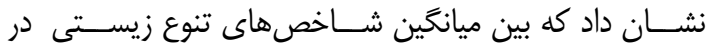

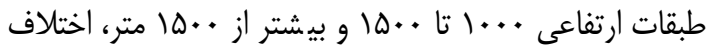

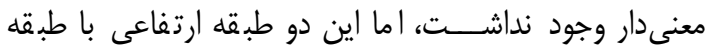

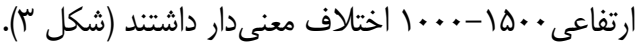

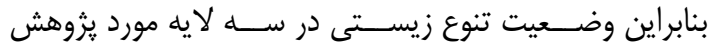

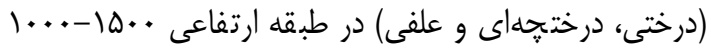

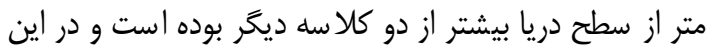

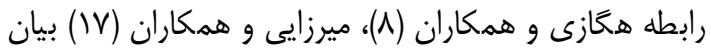

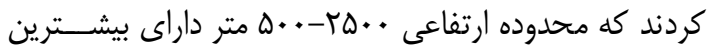

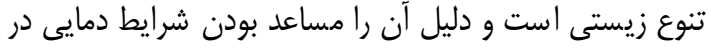




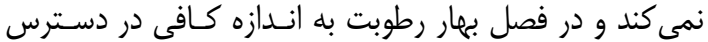

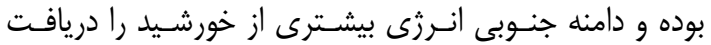

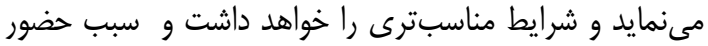

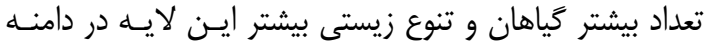

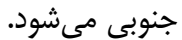
هدف اصلى از مديريت منان منابع طبيعى حفظ تنوع زيستى در

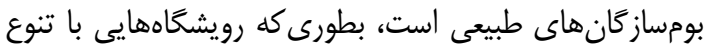

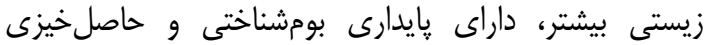

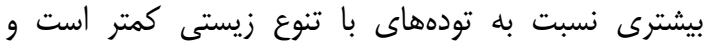

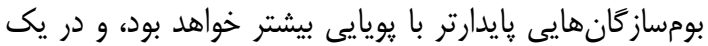

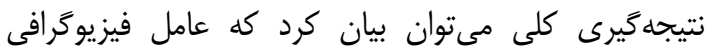

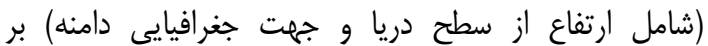

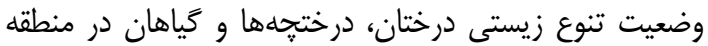

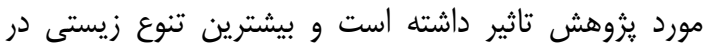

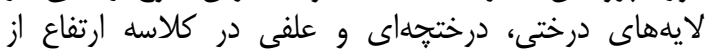

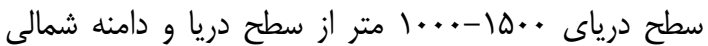
(غير از لايه علفى، كه تنوع بيشتر اين لايه در دامنه جنوبى دامي

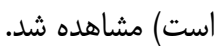

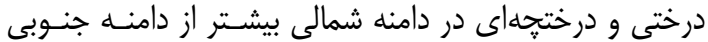

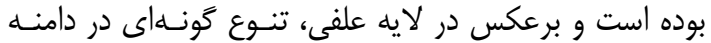

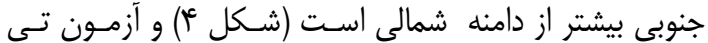

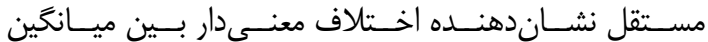

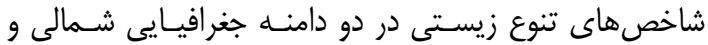

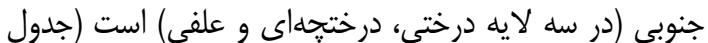

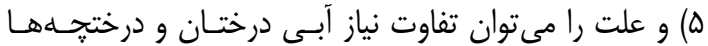

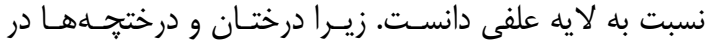

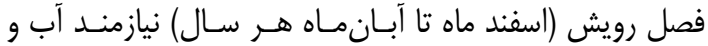

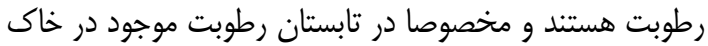

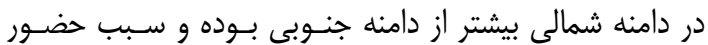

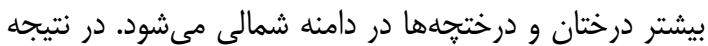

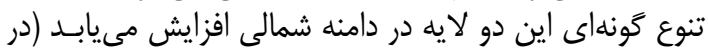

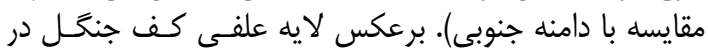

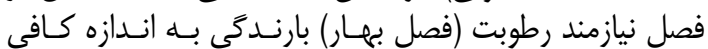

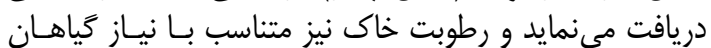

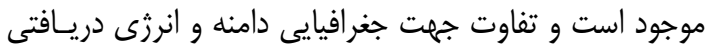

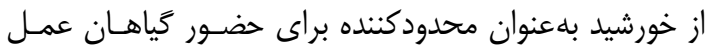
Abasi, S., B. Pilehvar and S.M. Hosseini. 2014. Study of plant biodiversity in Oshteranko protected area of Lorestan. Journal of Environmental Science and Technology, 16(3): 155-164 (In Persian).

2. Abd El-Wahab, R.H., A.R. Al-Rashed and A. Al-Dousari. 2018. Influences of Physiographic Factors, Vegetation Patterns and Human Impacts on Aeolian Landforms in Arid Environment. Arid Ecosystems, 8(2): 97-110.

3. Abdollahi, J., H. Naderi, A. Khavaninzadeh and M.S. Mahinifar. 2015. The relation between vegetation diversity and some of environmentally variables in Nodoushan steppe rangelands, Yazd. Journal of Range and Desert Research Iran, 22(2): 251-265.

4. Asri, U. 2006. Ecology of Vegetation Coverings. Payam Noor Publications. 209 pp (In Persian).

5. Barnes, B.V., D.R. Zak, S.R. Denton and S.H. Spurr. 1998. Forest Ecology. $4^{\text {th }}$ edn., John Wiley and Sons Inc., ISBN: 13: 978-0471308225773 pp

6. Boscutti, F., V. Casolo, P. Beraldo, E. Braidot, M. Zancani and C. Rixen. 2018. Shrub growth and plant diversity along an elevation gradient: Evidence of indirect effects of climate on alpine ecosystems. PLoS ONE 13(4): e0196653.

7. Hashemi, S.A. 2010. Evaluation plant species diversity and physiographical factors in natural broad leaf forest. American Journal of Environmental Sciences, 6(1): 20-25.

8. Hegazy, A.K., M.A. E1-Demedesh and H.A. Hosni. 1998. Vegetation, species diversity and floristic relations along an altitudinal gradient in south- west Saudi Arabi. Journal of Arid Environment, 3: 313.

9. Hosseini, A. 2016. Study effect of altitude gradient in diversity of tree species in oak forests (Heian Ilam). Iran's Natural Ecosystems, 7(1): 1-8 (In Persian).

10. Heidari, R.H. 2006. Study different inventory methods in Zagros forest (case study: Sorkhe Dizeh Rejion in Kermanshah). PhD Thesis in forestry science, University of Tehran, 112 pp (In Persian).

11. Heidari, M., S. Attar Roshan and Kh. Hatam. 2010. The evaluation of herb Layer biodiversity in relation to physiographical factors in south of Zagros forest ecosystem (case study: Dalab protected area). Journal of Renewable Natural Resources, 1(2): 28-42.

12. Jiang, Y., M. Kang, Y. Zhu and G. Ku. 2007. Plant biodiversity patterns on Helan Mountain, China. Acta Oecologica, 32: 125-133.

13. Jafari, J., M. Tabari, K. Kouchaksaraei, S.M. Hoseini and Y. Kooch. 2016. Effect of physiographical factors on plant species diversity in forests of west Bodjnourd. Journal of Wood \& Forest Science and Technology, 22(4): 2016 (In Persian).

14. Karami, R., H.R. Mehrabi and A. Ariapoor. 2015. The Effect of Altitude and Slope in the Species Diversity of Herbaceous Plants (Case Study: Watershed Miandar Qarootag - Gilangharb). Journal of Applied Environmental and Biological Sciences, 5(7): 197-204.

15. Khanhasani, M., Kh. Sagheb-Talebi, R. Akhavan and Zh. Vardanyan. 2015. The effect of environmental factors on distribution of three oak species (Quercus brantii Lindl., Quercus. libani Oliv. and Quercus infectoria Oliv.) in northern Zagros forests. Iranian Journal of Forest and Poplar Research, 23(3): 549-561 (In Persian). 
16. Mahmoudi, S., M. Khoramivafa and M. Hadidi. 2018. Investigation of the relationship between altitude and aspect with plant diversity: A case study from Nawa mountain ecosystem in Zagros, Iran. Journal of Rangeland Science, 8(2): 129-142.

17. Mirzaei, J., M. Akbarinia, S.M. Hosseini, H. Sohrabi and Hosseinzade. 2007. Biodiversity of herbaceous species in related to physiographic factors in forest ecosystems in central Zagros, Iranian Biology Journal, 20(4): 375-382 (In Persian).

18. Mirzaei, J., M. Akbarinia, S.M. Hosseni and M. Kohzadi. 2008. Biodiversity comparison of woody and ground vegetation species in relation to environmental factors in different aspects of zagros forest. Environmental Sciences, 5(3): 85-94 (In Persian).

19. Qian, H., R.E. Ricklefs and P.S. White. 2005. Beta diversity of angiosperms in temperate floras of eastern asia and eastern north america. Ecology Letters, 8: 15-22.

20. Smith, F. 1996. Biological diversity, ecosystem stability and economic development Journal of Ecological Economics, 16: 191-203.

21. Sekhavati, N., M. Akbarinia, H. Zanganeh and J. Mirzaee. 2016. Effect of topography on diversity of site in the forests of Kermanshah province. Forest and Rangeland, 97: 24-33(In Persian).

22. Tian, Z., W. Chenb, C. Zhaob, Y. Chenc and B. Zheng. 2007. Plant biodiversity and its conservation strategy in the inundation and resettlement districts of the Yangtze Three Gorges. China. Acta Oecologica Sinica, 27: 3110-3118.

23. Vujnovic, K., R.W. Wein and M.R.T. Dale. 2002. Predicting plant species diversity in response to disturbance magnitude in grassland remnants of central Alberta. Canadian Journal of Botany, 80: 504511.

24. Vaseghi, P., H. Ejtehadi and H. Zahedii pour. 2011. Plant Species Diversity in Relation to Elevation and Aspect: A case study in Kalat Highlands of Gonabad, Khorasan Razavi. Materials \& Energy, 9 (3): $547-558$ 


\title{
Effect of Physiographic Factors on Plant Biodiversity in the Central Zagros Forests (Case Study: Educational Forest of Razi University of Kermanshah)
}

\author{
Reza Hossaein Haidari ${ }^{1}$, Azadeh Sohrabi Zadeh ${ }^{2}$ and Maziar Haidari ${ }^{3}$
}

1 and 2- Assistant Professor and Graduate MSc., Faculty of Agriculture, Razi University, Kermanshah, Iran 3- Assistant Professor, Forests and Rangelands Research Department, Kurdistan Agricultural and Natural Resources Research and Education Center, AREEO, Sanandaj, Iran, (Corresponding Author: m.haidari@ areeo.ac.ir) Received: February 11, 2018 Accepted: December 12, 2018

\begin{abstract}
Ecological condition including physiographic factors have impact bon plant species diversity. There for it is necessary to investigate at the ecological condition in other to achieve sustainable development and natural ecosystem conservation. The present study aim is investigate the effect of physiographic factors (altitude and geographic directions) on the biological diversity of plant species in the middle Zagros forests. The investigate was conducted in Research and Education Forest at Razi university located in between the west cities of Kerend Gharb and Sarpole Zahab, in Kermanshah province. The studied area was divided into three elevation class including less than $1000 \mathrm{~m}, 1000$ to $1500 \mathrm{~m}$ and more than $1500 \mathrm{~m}$ asl. In each class of elevation and direction, respectively 32 and 48 plots of circular shapes of 1000 square meters in random- systematics were taken. In order to study the shrub layer in each circular sample plot, a plot of $25 \mathrm{~m}^{2}$ and a microplate of one square meter to study the herbaceous diversity were taken. The results showed that the highest values of diversity indices for tree, shrub and herbaceous cover in altitudes from (1500-1000) were observed and ANOVA test showed significant different between diversity index in three elevation classes. The results of compare biodiversity index in two main aspect showed that in trees and shrubs layer maximum of diversity index observed in the northern slopes, but for herbaceous layer, maximum of diversity index showed in southern slopes and T-test showed significant different of biodiversity index in two aspect direct. Overall results showed that the physiographical parameter (Elevation and aspect) are a significant effect on diversity condition.
\end{abstract}

Keywords: Biodiversity Indices, Elevation Class, Micro-Plots, Plot 